\title{
Breast cancer: initial workup and staging with FDG PET/CT
}

\author{
David Groheux ${ }^{1,2}$ (I) Elif Hindie ${ }^{3}$
}

Received: 7 February 2021 / Accepted: 13 April 2021 / Published online: 27 April 2021

(c) Italian Association of Nuclear Medicine and Molecular Imaging 2021

\begin{abstract}
Purpose Precise staging is needed to plan optimal management in breast cancer. 18F-fluorodeoxyglucose positron emission tomography coupled with computed tomography (FDG-PET/CT) offers high sensitivity in detecting extra axillary lymph nodes and distant metastases. This review aims to clarify in which groups of patients staging with FDG-PET/CT would be beneficial and should be offered. We also discuss how tumor biology and breast cancer subtypes should be taken into account when interpreting FDG-PET/CT scans.

Methods We performed a comprehensive literature review and rigorous appraisal of research studies assessing indications for FDG-PET/CT in breast cancer. This assessment regarding breast cancer served as a basis for the recommendations set by a working group of the French Society of Nuclear Medicine, in collaboration with oncological societies, for developing good clinical practice recommendations on the use of FDG-PET/CT in oncology.

Results FDG-PET/CT is useful for initial staging of breast cancer, independently of tumor phenotype (triple negative, luminal or HER2 +) and regardless of tumor grade. Considering histological subtype, FDG-PET/CT performs better for staging invasive ductal carcinoma, although it is also helpful for staging invasive lobular carcinomas. Based on the available data, FDG-PET/CT becomes useful for staging starting from clinical stage IIB. FDG-PET/CT is possibly useful in patients with clinical stage IIA (T1N1 or T2N0), but there is not enough strong data to recommend routine use in this subgroup. For clinical stage I (T1N0) patients, staging with FDG-PET/CT offers no added value.
\end{abstract}

Conclusion FDG-PET/CT is useful for staging patients with breast cancer, starting from clinical stage IIB.

Keywords Breast cancer $\cdot$ FDG $\cdot$ PET/CT $\cdot$ Staging $\cdot$ Initial workup

\section{Introduction}

Breast cancer (BC) is the most frequent type of cancer in women. In the United States, 281,550 new cases of invasive $\mathrm{BC}$ and 43,600 deaths in women are expected in 2021[1]. Several treatment weapons (surgery, radiation therapy, chemotherapy, targeted therapy, endocrine therapy) are available. To use optimal treatment for a given patient, however, the biological characteristics of the tumor and the precise staging of the disease need to be known. In particular,

David Groheux

dgroheux@yahoo.fr

1 Department of Nuclear Medicine, Saint-Louis Hospital, Paris, France

2 University Paris-Diderot, INSERM U976, HIPI, Paris, France

3 Department of Nuclear Medicine, Bordeaux University Hospital, Bordeaux, France the presence or absence of distant metastases should be assessed.

During the last years, 18F-fluorodeoxyglucose positron emission tomography coupled with computed tomography (FDG-PET/CT) has gained an increasing role in the pretreatment staging of breast cancer. FDG-PET/CT has shown high accuracy in detecting extra axillary lymph nodes and distant metastases. Given the high incidence of $\mathrm{BC}$, costs of FDG-PET/CT imaging, radiation exposure, and the inconvenience associated with possible false-positive findings, it is important to determine in which groups of patients staging with FDG-PET/CT would be beneficial and should be offered.

The work of several teams has now allowed to clarify the situations in which FDG-PET/CT would be most helpful as well as pointing limitations encountered in specific situations. We summarize the most salient data up to December 2020 and report conclusions on the role of FDG-PET/CT in breast cancer. 


\section{Literature search and methodology used for assessing the utility of FDG-PET/CT in various situations at initial staging}

Recently, the French Society of Nuclear Medicine (SFMN), in collaboration with oncological societies, set up a focus group to work on developing good clinical practice recommendations for the use of PET/CT in oncology. A comprehensive literature review and rigorous appraisal by a panel of experts, organ specialists, clinical oncologists, surgeons and imaging specialists has been undertaken [2]. These good clinical practice recommendations have been awarded joint French National Health Authority (HAS) and French Cancer Institute (INCa) label status. Summary statements of the recommendations have been published in the European Journal of Nuclear Medicine and Molecular Imaging [2].

Having been in charge of examining the data related to breast cancer, we here discuss the arguments that made it possible to carry out the recommendations for the use of FDG-PET/CT in breast cancer. The literature search strategy in terms of completeness of screening and data extraction followed the methodology established by the HAS [2].

The method for developing the recommendations was based on: (1) critical analysis of the best available scientific data to attribute a level of evidence to conclusions drawn from the literature; and (2) the reasoned opinion of the working group experts. The recommendations were formulated by the multidisciplinary working group based on the level of evidence as defined by HAS criteria [2]. The recommendations were additionally reviewed by an independent panel of experts before being finally adopted. The recommendations formulated are graded with two levels of strength:- 'PET/CT is recommended' means that the imaging modality is unanimously recognized by the experts as the clinical reference standard.-if a clinical service was found to be acceptable on the basis of literature data or expert opinion but not unanimously recognized as the clinical reference standard, the text states that 'PET/CT can be proposed'.

More specifically for breast cancer, the search of literature (with Medline ${ }^{\circledR}$ as the main database) used the following criteria: adult population, breast cancer, FDG-PET or PET/CT. The oncological situations covered included (cancer diagnosis and tumor characterization, assessment of multifocality and T score, assessment of axillary status, staging, prognostic value, restaging for suspected recurrence, follow-up, treatment response evaluation). The studies were selected if "meta-analysis" or "prospective study" or "retrospective study" judged of sufficient quality and offering the necessary information regarding test performance: sensitivity, specificity, positive predictive value, negative predictive value. We excluded case reports. The literature search and selection process finally retained 157 references, that served for the issued recommendations [2]. For completeness of the present review article, the literature search was updated to also include all relevant recent articles published until Dec. 30, 2020. The level of agreement of the findings from recent studies with the issued recommendations is also discussed. In the present rewiew, 70 relevant articles are cited.

\section{Preamble: Parameters affecting FDG uptake in breast cancer tumors}

Appropriate interpretation of FDG-PET/CT exams requires sharp knowledge of potential false negatives, which might result from small tumor size (partial volume effect) or low FDG uptake. The main factors influencing tumoral uptake are as follows:

- Tumor grade: according to the Elston-Ellis modification of the Scarff-Bloom-Richardson (SBR) grading system, grades 1 or 2 tumors show lower FDG uptake than grade 3 tumors [3].

- Histological subtype: FDG avidity is lower for invasive lobular carcinomas than invasive ductal carcinomas [3]. Ductal carcinoma in situ (DCIS) usually show lower uptake compared with invasive carcinomas.

- Proliferation index: FDG uptake is weaker in low-proliferative tumors as assessed by the Ki67 index [4, 5].

- p53 status: FDG uptake is higher in tumors with mutated p53 [3].

- Hormone receptor status: FDG uptake is lower in welldifferentiated estrogen receptor (ER)-positive tumors than ER-negative tumors. This is also the case for progesterone receptor (PR)-positive tumors compared with PR-negative tumors [3].

- Tumor phenotype: triple-negative tumors i.e., ER negative, PR negative and having no overexpression of HER2 (ERBB2) show substantially higher SUVs than other tumors. Among luminal tumors, FDG uptake is lower in luminal A tumors than in luminal B tumors [6].

\section{Diagnosis of breast cancer and tumor characterization}

More than twenty studies have evaluated the role of PET imaging with FDG (PET or PET/CT systems) for the characterization (benign/malignant differentiation) of a breast tumor. The oldest publications have shown encouraging results in the detection of primary malignant tumor [7-9]. These early studies involved small populations and patients 
with large breast lesions. The meta-analysis by Sanson et al. published in 2002 included data from 13 studies (606 patients) and made it possible to estimate the sensitivity of PET at $89 \%$ and its specificity at $80 \%$ [10]. However, more recent studies with higher proportions of subcentimeter tumors has shown poorer performance [11-13]. In the study by Kumar et al. [12], among 111 women, FDG PET could detect only 41 of the 85 malignant tumors, ie a sensitivity of $48 \%$. The main causes of false negatives were small size $(\leq 10 \mathrm{~mm})$ and low tumor grade [12]. Thus, limited sensitivity is explained by limited spatial resolution of PET imaging for small subcentimeter, as well as some characteristics of the tumor, for example weak FDG uptake in the case of lobular carcinoma, low-grade tumor, or luminal-A tumor $[12,14]$.

The specificity is also limited. Some benign tumors, such as fibroadenomas can have significant FDG uptake [15]. Uptake is usually intense in case of breast infectious or inflammatory processes [16]. To improve specificity, some authors recommend recording late images, as uptake would usually increase on delayed imaging in case of malignancy, while it often decreases in inflammatory lesions [17]. However, this method is time consuming, and its usefulness has not been validated on large series.

PET systems dedicated to breast imaging, allowing positron emission mammography (PEM) have higher detection performance than "whole body" PET imaging, especially with regard to sensitivity [18-20]. The meta-analysis by Caldarella et al. evaluated eight studies including 873 women with breast lesions. The sensitivity and specificity values of PEM in case of suspected breast cancer were $85 \%$ (95\% CI 83-88\%) and 79\% (95\% CI 74-83\%), respectively [20]. The included studies were heterogeneous in their estimation of sensitivity and specificity. These systems have not been evaluated on a large scale and are not widely available in Europe.

Hybrid PET-MRI imaging has also shown interesting performances [21]. In a group of 36 patients, the 37 known index cancers were detected by PET and MRI [22].

Incidental breast lesions on FDG-PET examinations: among 4038 patients receiving FDG-PET/CT (for a reason other than breast cancer), an FDG-avid breast lesion was fortuitously discovered in 33 patients $(0.82 \%)$; focal uptake corresponded to a malignant lesion in $57 \%$ of patients [23].

In conclusion, FDG-PET/CT has low sensitivity and specificity for informing on the malignancy of a breast lesion. FDG-PET/CT cannot substitute for biopsy in determining the malignancy of a breast lesion. When an FDGavid focus is seen in the breast during an FDG-PET/CT exam performed for other reasons, the possibility of a malignant breast lesion cannot be neglected and should be considered.

The following recommendations have been made [2]:
- FDG-PET/CT is not recommended for characterizing a breast lesion as "diagnosing malignancy".

- When FDG exams performed for other reasons come up with an incidental finding of an FDG-avid intra-mammary focus, it is recommended to pursue investigations, even though some benign lesions such as fibroadenomas may give false-positive uptake.

\section{Assessment of breast cancer multifocality and T status (in TNM staging)}

Few studies have analyzed the role of PET/CT in determining the T score of the TNM classification [24]. Due to its limited spatial resolution, PET is less sensitive and less accurate than MRI for delineating the volume of the primary tumor and for assessing for multifocality $[13,25]$. In a group of 40 women undergoing PET/CT and MRI [25], MRI assessed the T classification correctly in $77 \%$ of cases and PET/CT in only $54 \%$ of cases $(p=0.001)$.

Better performances are expected with high-resolution PEM imaging [20], as well as with PET/MRI imaging [21, 22]. In a group of 36 patients, the 37 known index cancers were detected by PET and MRI; 47 satellite lesions were additionally detected on MRI, of which 23 were avid for FDG, with a multifocal/multicentric character in $21(58 \%)$ patients [22].

In conclusion, FDG-PET/CT performs sub-optimally on delimitating primary tumor volume and assessing for multifocality; MRI offers greater sensitivity. There is still not enough good data on breast-dedicated PEM systems and on PET-MRI to conclude on their performances for assessing multifocality or determining the $\mathrm{T}$ status of a breast tumor.

The following recommendations could be made [2]:

- FDG-PET/CT is currently not recommended for assessing multifocality or assessing the $\mathrm{T}$ status of a breast tumor.

- In the future, in the context of multi-centric and randomized studies, PEM and PET-MRI could be evaluated to search the multifocality of known breast cancer and to assess the $\mathrm{T}$ score.

\section{FDG-PET/CT compared with sentinel lymph node biopsy for determining axillary status}

The spatial resolution of PET is limited and hence sensitivity is low for small lymph node metastases and micrometastases [26-30]. A prospective multicenter study investigated PET scan (without CT component) in 360 women with newly diagnosed invasive breast cancer [26]. For the detection of axillary lymph node metastases, the 
mean sensitivity and specificity of PET were $61 \%$ and $80 \%$, respectively [26]. In the prospective study by Veronesi et al., 236 patients without palpable nodes underwent FDG-PET and sentinel lymph node biopsy; only $37 \%$ of positive sentinel nodes were detected by PET [27]. A meta-analysis evaluated the performance of PET (with or without CT) for depicting axillary involvement [30]. Among 19 studies with stand-alone PET (1729 patients), the sensitivity was $66 \%(50-79 \%)$ and the specificity, $93 \%$ (89-96\%). Among seven studies based on PET/CT (862 women), sensitivity was $56 \%(44-67 \%)$ and specificity 96\% (90-99\%) [30]. For assessing axillary status, PET/ CT does not appear to be superior to ultrasound [31] or MRI [32].

Nevertheless, the presence of FDG-avid axillary foci is suggestive of malignancy with a positive predictive value exceeding $80 \%$ in most studies [27, 33].
In conclusion, the spatial resolution of PET imaging is insufficient for depicting small axillary node metastases.

The following recommendations have been made [2]:

- FDG-PET/CT is not recommended to replace sentinel lymph node biopsy.

\section{Initial workup and assessment of $\mathbf{N}$ and $M$ status in the TNM-AJCC "anatomic" classification}

FDG-PET/CT has shown high performance for the pretreatment workup of inflammatory and locally advanced breast cancer [16, 34-38] (Figs. 1, 2). A recent systematic review showed that FDG-PET/CT outperforms conventional imaging procedures for the detection of locoregional and

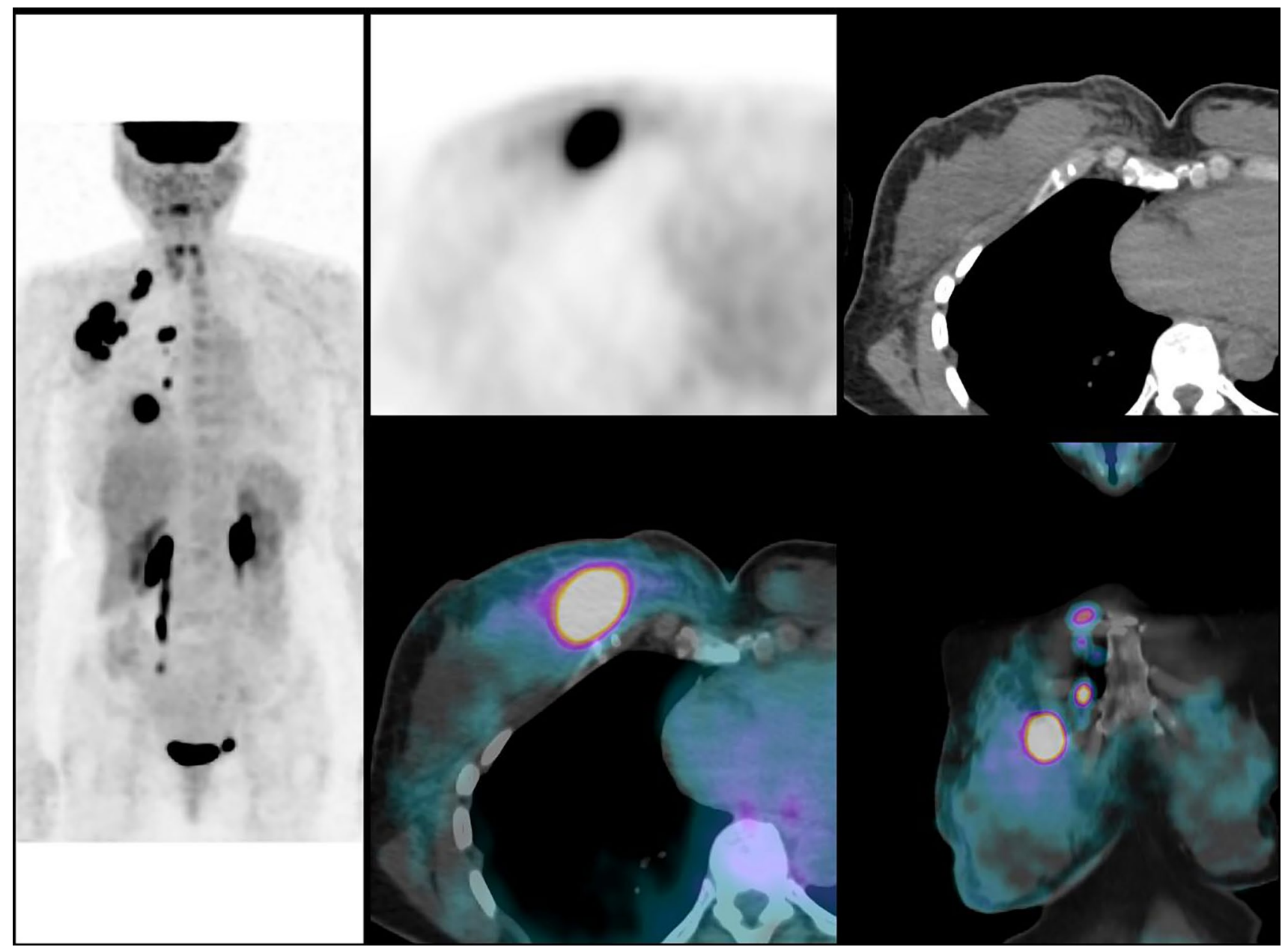

Fig. 1 A 54 years, menopaused woman with an invasive ductal carcinoma of the right breast, grade 3, ER-, PR-, HER2 + . Examination and palpation show a mass of $80 \mathrm{~mm}$, with inflammation of the skin, fixed axillary lymph nodes metastasis and no supraclavicular lymph nodes: TNM classification before PET/CT is cT4d cN2a cM0 (stage
IIIB). PET/CT shows the primary breast cancer with axillary lymph nodes (level I, II and III), supra-clavicular and internal mammary lymph nodes: TNM classification after PET/CT is T4 N3c M0 (stage IIIC). PET/CT fusion images show FDG uptake in the primary tumor and in internal mammary lymph nodes 


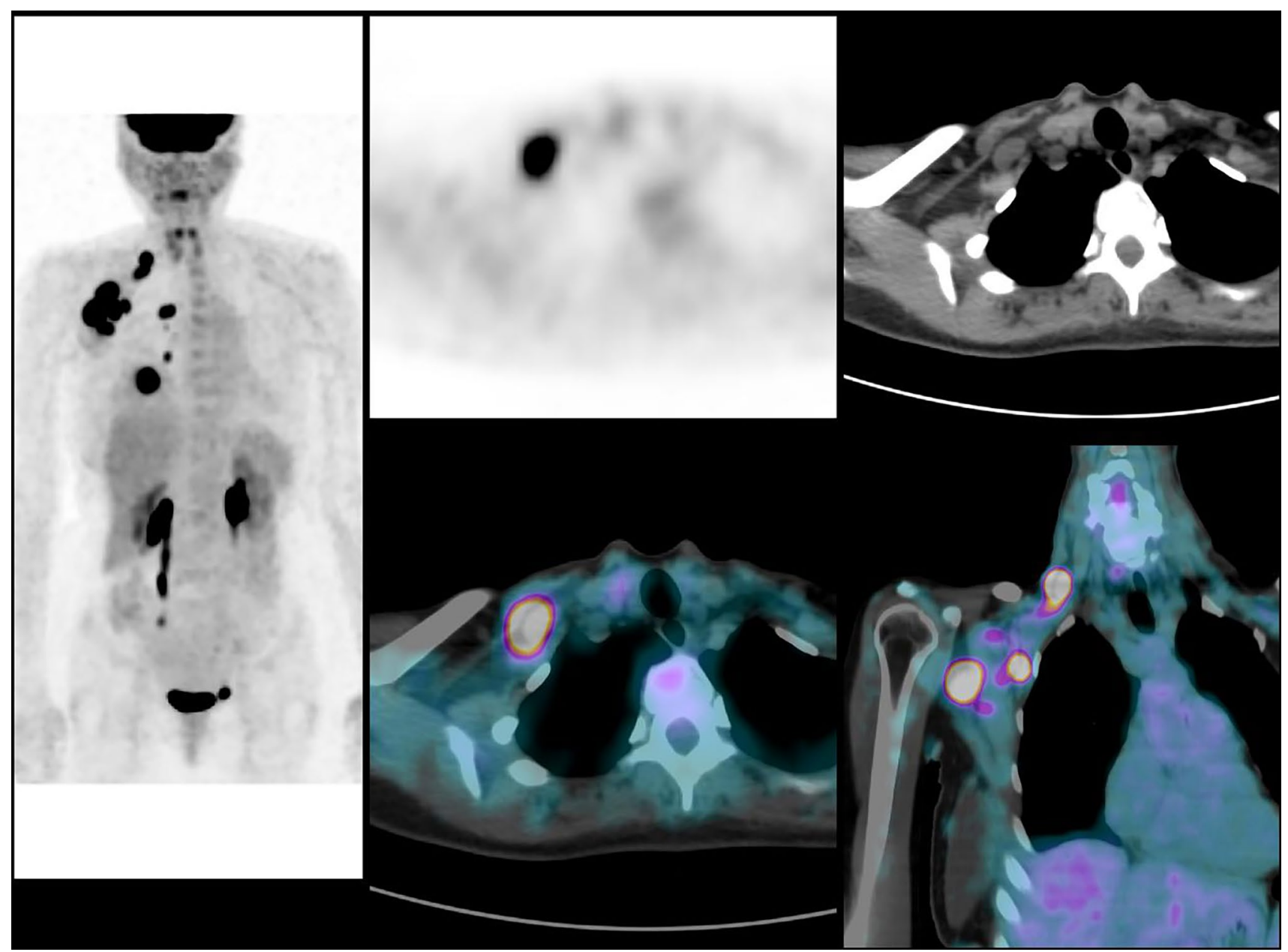

Fig. 2 Same patient as seen in Fig. 1. PET/CT fusion images show numerous hypermetabolic lymph nodes in the axillary area (level I to level III) and in the supra-clavicular area

distant metastases in the diagnostic workup of inflammatory breast cancer patients [39]. The definition of locally advanced breast cancer varies somewhat in the literature. According to the National Comprehensive Cancer Network (NCCN), it corresponds to American Joint Committee on Cancer (AJCC) stages IIIC, IIIB and IIIA (except for T3N1 tumors). $\mathrm{LABC}$ have thus at least one of the following: $\mathrm{T} 4$ or N2 or N3 [24, 40, 41] (Table 1). Several studies have also highlighted the role of FDG-PET in less advanced stages (Fig. 3) [42-53].

\section{Contribution of PET/CT in the assessment of extension of breast cancer}

FDG-PET/CT allows on a single "whole-body" examination to assess for locoregional as well as distant metastases [42, 43, 46, 48].

With the CT component of hybrid PET/CT imaging, it is possible to distinguish involved lymph nodes located at axillary Berg level III (infra-clavicular area, supero-medial to the pectoralis minor muscle), from those located in the lower axillary level I and II $[48,54]$. This information is useful because dissection of axillary lymph nodes is usually limited to levels I and II. PET can also assess for extra-axillary regional node disease (supraclavicular area and internal mammary chain) (Figs. 1, 2) [48, 54].

Based on available data, the positive predictive value (PPV) of FDG-PET/CT for foci identified in usual drainage areas of $\mathrm{BC}$ is rather high $[55,56]$. In our prospective study, we could also confirm the high PPV of FDG-PET/ CT [38]. This might justify RT on the basis of FDG finding when biopsy is difficult to obtain. The possibility of falsepositive should be considered, however, notably if imaging is performed in the post-operative setting. The possibility of inflammatory nodes due to recent vaccination against Covid-19 should also be in mind [57]. 
Table 1 TNM Stage grouping for breast cancer according to the AJCC Cancer Staging Manual [24]

\begin{tabular}{lllll}
\hline AJCC & TNM & \multicolumn{2}{c}{ NCCN } \\
\hline Stage I & T1 & N0 & M0 & Primary operable breast cancer \\
Stage IIA & T0 & N1 & M0 & \\
& T1 & N1 & M0 & \\
& T2 & N0 & M0 & \\
Stage IIB & T2 & N1 & M0 & \\
& T3 & N0 & M0 & \\
Stage IIIA & T3 & N1 & M0 & \\
& T0 & N2 & M0 & Locally advanced breast cancer \\
& T1 & N2 & M0 & \\
& T2 & N2 & M0 & \\
& T3 & N2 & M0 & \\
Stage IIIB & T4 & N0 & M0 & \\
& T4 & N1 & M0 & \\
& T4 & N2 & M0 & \\
Stage IIIC & Any T & N3 & M0 & \\
Stage IV & Any T & Any N & M1 & Metastatic disease \\
\hline
\end{tabular}

PET/CT is very effective in detecting occult distant metastases (except for brain), with performances superior to those of conventional imaging.

$\mathrm{PET} / \mathrm{CT}$ is more sensitive and more specific than contrast-enhancement CT or bone scan to detect lytic or mixed bone metastases, or bone marrow involvement [42, 48, 58]; FDG uptake is more variable in osteoblastic metastases, and careful reading of the CT-scan data from PET/ CT can help detect them [54]. In a study of 23 breast cancer patients with bone metastases, PET/CT detected more lesions than bone scintigraphy (mean 14.1 vs. 7.8 lesions, respectively, $P<0.01$ ) [59]. However, in the subgroup of patients with osteoblastic lesions, FDG-PET/CT showed less metastases than bone scintigraphy $(P<0.05)$. Higher SUV values were observed for osteolytic lesions compared to osteoblastic lesions (mean: 6.77 vs. 0.95 , respectively, $P<0.01)$. Survival was lower in patients with osteolytic disease compared to others $(P=0.01)$ [59]. Also, osteoblastic lesions with no FDG uptake had a better prognosis [59].

Several other studies have shown that bone scintigraphy is not useful when PET/CT is performed [38, 48, 58]. In a group of 163 women with breast cancer, results of PET/CT and bone scintigraphy were concordant in 132 cases (both examinations were positive in 32 women and both were negative in 100 women) [58]. Among 31 women (19\%) with discordant results, 12 had a biopsy confirming bone metastases: 9 cases were PET/CT positive and bone scintigraphy negative, 1 was PET/CT positive and bone scintigraphy equivocal and the last 2 were PET/CT equivocal and bone scan negative [58].
$\mathrm{PET} / \mathrm{CT}$ is also quite effective in detecting extra-skeletal metastases, including, distant nodal disease, pleural, hepatic, splenic, adrenal, and pelvic metastases [42-52]. In a group of 117 patients with locally advanced breast cancer, PET/ CT enabled the detection of distant metastases in 43 patients (37\%) [38]; the metastatic sites were: bone $(n=30)$, distant lymph nodes $(n=19)$, liver $(n=10)$, lungs $(n=6)$ and pleura $(n=2)$. The sensitivity, specificity, positive predictive value, negative predictive value and accuracy for the diagnosis of bone lesions were $76.7 \%, 94.2 \%, 82.1 \%, 92.1 \%$ and $89,7 \%$ respectively for planar bone scintigraphy and $100 \%, 97.7 \%$, 93.7\%, 100\% and 98.3\% for FDG-PET/CT [38]. The sensitivity, specificity, positive predictive value, negative predictive value and accuracy for the diagnosis of pulmonary metastases were respectively $100 \%, 98.2 \%, 77.8 \%, 100 \%$ and $98.3 \%$ for dedicated chest CT and $85.7 \%, 98.2 \%, 75 \%$, $99.1 \%$ and $97.4 \%$ for PET/CT. In this study, therefore, PET was less sensitive than a chest CT scan to detect small lung nodules, which could be explained by the partial volume effect and respiratory motion [38]. The sensitivity, specificity, positive predictive value, negative predictive value and accuracy for the diagnosis of pleural metastases were respectively $50 \%, 100 \%, 100 \%, 99.1 \%$ and $99.1 \%$ for the dedicated CT and $100 \%, 99.1 \%, 66.7 \%, 100 \%$ and $99.1 \%$ for FDG-PET/CT. Regarding distant lymph node involvement, 18 patients had supra diaphragmatic involvement (cervical, mediastinal, hilar or contralateral axillary lymph nodes) on PET/CT and four patients had FDG uptake in abdominal and/or pelvic lymph nodes [38]. Out of the 117 patients, 10 had liver metastases. PET/CT depicted all nine cases identified by abdominal CT and/or liver ultrasound as well as one additional patient [38]. Moreover, in 3 other patients, PET/ CT made it possible to clarify doubtful images of conventional imaging [38]. In one patient, hepatic ultrasound suggested metastases (false positive). A second patient had a biliary cyst and the last patient had a hepatic angioma; these lesions had no FDG uptake [38].

In another study, including 60 patients, the sensitivity and specificity of PET/CT to detect distant metastases were $100 \%$ and $98 \%$, respectively (vs. $60 \%$ and $83 \%$ for conventional imaging) [43]. PET/CT allowed to detect hepatic metastases in two patients [43]. The liver ultrasound showed suspicious lesions in five women, which were ultimately benign (three angiomas and two cysts) [43].

In another recent study, of 103 women, 24 (23\%) were diagnosed with distant metastases by FDG-PET/CT [60]. Based on these findings, breast surgery was omitted in 18. Another sixteen patients (16\%) were upstaged to more advanced loco-regional disease, leading to more extensive radiotherapy. Sensitivity and specificity for diagnosing distant metastases were 1.00 (95\% confidence interval: $0.86-1.00)$ and $0.95(0.88-0.99)$, respectively. Twentynine incidental findings were detected in 24 women (23\%), 


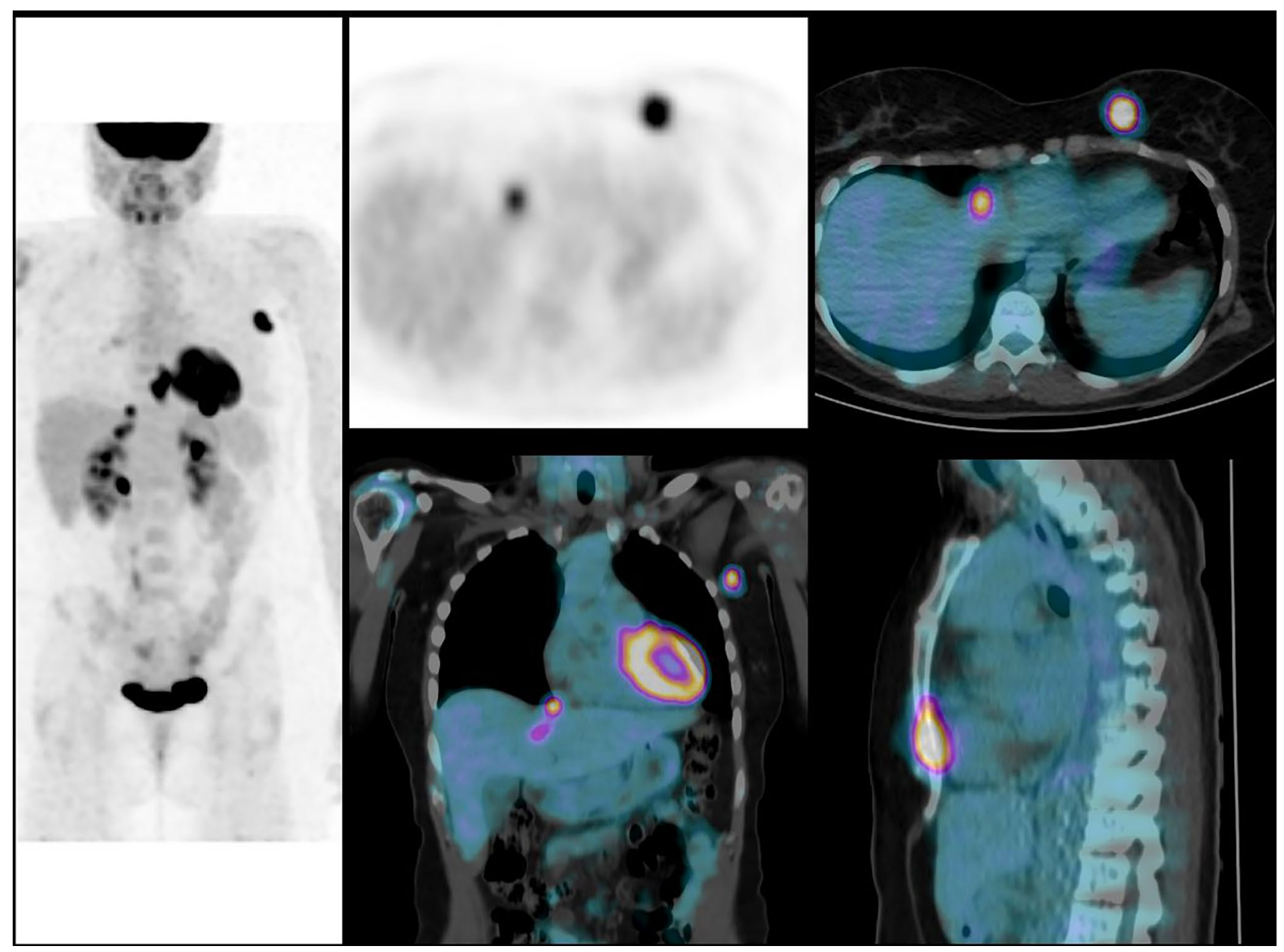

Fig. 3 Patient with a 52-mm invasive ductal carcinoma of left breast and movable axillary lymph node: TNM classification before PET/CT is cT3 cN1 cM0 (stage IIIA). FDG-PET/CT shows high FDG uptake

leading to further examinations in 22 and diagnosis of eight $(8 / 22,36 \%)$ synchronous diseases: cancer $(n=4)$, thyroiditis $(n=2)$, aorta aneurysm $(n=1)$, and meningioma $(n=1)$. The authors concluded that FDG-PET/CT should be considered for primary staging in high-risk primary breast cancer to improve treatment planning [60].

\section{Impact of tumor biological characteristics on results of FDG-PET/CT distant workup}

As discussed previously, besides small tumor size, the main factors limiting sensitivity of FDG breast cancer imaging are low tumor grade, low proliferation, high expression of hormone receptors (especially luminal A phenotype), and lobular histologic type [3].

In a prospective study of 254 patients at Saint-Louis Hospital in Paris, Groheux et al. investigated whether the number of metastases detected with PET/CT differed according to the grade or subtype of the primary tumor [48]. The rates in primary tumor, axillary lymph node, and distant metastases to the sternum and liver: TNM classification after PET/CT is T3 N1 M1 (stage IV). Treatment was adapted to metastatic disease

of extra-axillary lymph node metastases on FDG-PET/CT were higher in grade- 3 than in low-grade tumors $(P=0.004)$ and in triple negative or HER $2+$ tumors compared to ER + 1 HER2- tumors $(P=0.01)$. The rate of distant metastases, however, was not related to tumor grade or breast cancer subtype [48], a finding which was also confirmed by other studies [51, 61]. Let us note, however, that the distribution of the lesions differed according to the breast cancer subtype; triple negative and HER2 + tumors exhibited more extra-skeletal metastases than ER +/HER2- tumors [48]. In addition, two studies by the Memorial Sloan-Kettering Cancer Center (MSKCC) team showed limited performance of FDG-PET for the workup of the lobular histological type, but analysis of CT images of the PET/CT examination helped to detect lesions with faint or no FDG uptake [62, 63]. Among 146 patients with infiltrating lobular carcinoma, PET/CT revealed distant metastases (confirmed by biopsy) in 12 cases; in 3 of these 12 patients, metastases had no FDG uptake and were seen only on the CT component of the 
examination [63]. When considering patients with a similar clinical stage (stage III), the yield of FDG-PET/CT for distant metastases, leading to stage IV, was lower in invasive lobular (11\%) than in invasive ductal (22\%) carcinoma [63].

\section{The yield of FDG-PET/CT for initial workup according to the clinical stage of breast cancer}

Patients with a small tumor $\leq 2 \mathrm{~cm}$ (T1 of the TNM classification) are usually treated with primary surgery associated with sentinel node biopsy. PET has limited spatial resolution (around 5-6 $\mathrm{mm}$ ) and its performance is inferior to the sentinel node biopsy [30].

In addition, the risk of distant metastases in T1 N0 disease (stage I of AJCC) is very low. In patients with stage I breast cancer, the workup could delay therapeutic management and/or cause unnecessary anxiety. In a multicenter study of 325 women with operable breast cancer, FDG PET scans (without CT component) suggested distant metastases in 13 patients; only $3(0.9 \%)$ were confirmed as metastatic disease and $10(3.0 \%)$ were false positives [28].

Between locally advanced breast cancer, where the role of FDG-PET/CT is uniformly admitted, and T1 N0 disease where its role is recognized as futile, there are several intermediaterisk stages (IIA, IIB and T3N1 disease of stage IIIA) (Fig. 3). For many of these patients, and notably so in aggressive tumor subtypes, treatment now begins with neoadjuvant chemotherapy to increase the chance of breast-conserving surgery.

In the previously mentioned prospective study on 254 patients with breast cancer $>2 \mathrm{~cm}$, Groheux et al. assessed the role of FDG-PET/CT in relation to the clinical stage before starting neoadjuvant chemotherapy [48]. The initial clinical stage (IIA, IIB, IIIA, IIIB, and IIIC) was determined by clinical examination, mammography, breast MRI, and locoregional ultrasound. PET/CT led to a change in breast cancer staging in 77 of 254 patients (30.3\%). It showed unsuspected N3 disease (sub- or supra-clavicular or internal mammary nodes) in 40 women and distant metastases in 53 women [48]. Distant metastases were detected in 2.3\% (1/44) of patients with clinical stage IIA cancer; in $10.7 \%$ (6/56) patients with stage IIB; in $17.5 \%$ (11/63) patients with stage IIIA; in 36.5\% (27/74) patients with stage IIIB and in 47.1\% (8/17) patients with stage IIIC breast cancer. Stage IIIA is heterogeneous. The rate of occult metastases in $\mathrm{T} 3 \mathrm{~N} 1$ cancer was similar to stage IIB (T2N1 / T3N0). The number of metastatic patients was higher for patients with $\mathrm{N} 2$ disease, close to that seen for stage IIIB [48]. Several other studies have also shown that PET/CT provides significant diagnostic benefit in patients treated for clinical stage IIB breast cancer [44, 51, 61, 64]. The yield in clinical stage IIA is less established. In the retrospective study by Lebon and colleagues, distant metastases were detected by FDG-PET/CT in 15\% of stage IIB patients, and in $11 \%$ of stage IIA patients [65]. In another recent retrospective study, however, PET/CT demonstrated distant disease in 9.8\% of stage IIB breast cancer patients, but in only $0.8 \%$ of those with stage IIA [66].

In the study by Groheux et al., Stage IIA was mainly represented by tumors classified as T2N0. PET showed pathological foci in $4.5 \%$ of women ( $2.3 \%$ distant metastases and $2.3 \%$ extra-axillary nodes) [48]. Larger studies in which the performance of PET is examined in subcategories of patients with $\mathrm{T} 2 \mathrm{~N} 0$ disease, such as those with large tumors $(\mathrm{T} 2>3 \mathrm{~cm})$ would be useful. In patients with clinically negative axilla, the possibility of internal mammary (IM) node involvement also deserves discussion, notably for tumors in the internal quadrants. Imaging techniques for detecting IM node metastasis include ultrasound (a suspicious node may be considered for ultrasound-guided fine-needle aspiration), computed tomography, magnetic resonance imaging, and FDG-PET/CT [67]. None of them, however, has enough sensitivity for detecting micrometastases. The probability of FDG-PET/CT identifying occult IM disease in patients with T2N0 tumors is low; being $2.3 \%$ in our prospective study [48]. Drainage to IM chain is seen in about $20 \%$ of patients on sentinel lymph node scintigraphy when the radiotracer is injected peritumorally [68]. In the presence of IM drainage, the risk of IM involvement depends upon various factors, notably the status of axillary sentinel lymph node [68].

In addition, patients with T2N0 disease are often treated with neoadjuvant chemotherapy, and in this case pre-therapeutic PET could be used to perform an early assessment of therapeutic response. Please also note that in our study [48], axillary node detection was not considered a finding as surgery with axillary dissection after neoadjuvant therapy was routine at the time. The situation is now different as information on axillary status before starting neoadjuvant therapy can be helpful for decision-making regarding axilla management post-NAC.

At this time, NCCN guidelines do not recommend FDGPET/CT but it is stated "FDG-PET/CT may be helpful in identifying unsuspected regional nodal disease and/or distant metastases in locally advanced breast cancer when used in addition to standard imaging studies" [40]. This may evolve, however. In a recent study reported in the JNCCN [69], among 196 breast cancer patients, the overall upstaging rate to stage IV based on findings of unsuspected distant metastases was $14 \%$ (27/196), including $0 \%$ for stage IIA, $13 \%$ for stage IIB (10/79), $22 \%$ for stage IIIA (9/41), 17\% for stage IIIB (5/30), and $37 \%$ for stage IIIC (3/8). PET/CT had comparable costs than conventional imaging, consisting in contrast enhanced CT plus bone scan, and had lower radiation dose exposure [69]. In another recent multicenter study, PET/CT reduced false-positive risk by half and decreased work-up for incidental findings, allowing for earlier treatment start. PET/CT was cost-effective, and at one institution was shown to be costsaving [70]. These two recent manuscripts in JNCCN add 
Table 2 Summary of the recommendations regarding the use of FDG PET/CT at initial workup of breast cancer

$\begin{array}{lll}\text { FDG-PET/CT } & \text { FDG-PET/ } & \text { FDG-PET/CT Not recommended } \\ \text { Recommended } & \text { CT Optional } & \end{array}$

Characterizing a breast lesion "diagnosing malignancy"

Assessing multifocality, or assessing the T status of a breast tumor

Determining axilla status (in replacement of sentinel lymph node biopsy)

Staging patients with clinical stage I (T1N0) breast cancer

Staging patients with clinical stage IIA (T1N1 or T2N0) breast cancer

Staging breast cancer patients with clinical stage IIB or higher
Not recommended

Currently not recommended ${ }^{\mathrm{a}}$

Not recommended

Not recommended

Optional

Recommended $^{\mathrm{b}}$

${ }^{a}$ This recommendation relates to current whole-body PET-CT examination. The specific role of PEM and PET-MRI in assessing multifocality and $\mathrm{T}$ score deserves investigation

${ }^{b}$ FDG-PET/CT is useful for initial staging in these patients regardless of tumor grade or tumor phenotype (triple negative, luminal or HER2 +). Considering histological subtype, FDG-PET/CT performs better in invasive ductal carcinoma, but it is also helpful for staging invasive lobular carcinomas

financial and radiation protection data to support the assertion that FDG-PET/CT should be utilized in patients with stage IIB-IIIC breast cancer. We hope this growing evidence will lead NCCN guidelines to include FDG-PET/CT for the systemic staging of IIB-IIIC breast cancer at initial diagnosis.

In conclusion, FDG-PET/CT is useful for the initial staging of breast cancer, independently of tumor phenotype (triple negative, luminal or HER2 +) and regardless of tumor grade. Considering histological subtype, FDG-PET/CT performs better for staging invasive ductal carcinoma (invasive carcinoma of no specific subtype), although it is also helpful for staging invasive lobular carcinomas.

Based on the available data, FDG-PET/CT becomes useful for staging starting from clinical-stage IIB. FDG-PET/CT is possibly useful in patients with clinical stage IIA (T1N1 or T2N0), but there is not enough strong data to recommend routine use in this subgroup. For clinical stage I (T1N0) patients, staging with FDG-PET/CT offers no added value.

These findings justify the following recommendations [2] (Table 2):

- FDG-PET/CT is recommended for initial staging in patients with clinical stage $\geq$ IIB breast cancer and is better when performed before surgery.

- FDG-PET/CT can be proposed for staging patients with clinical stage IIA (T1N1 or T2N0) breast cancer and is better when performed before surgery.

- FDG-PET/CT is not recommended for staging patients with clinical stage I (T1N0) breast cancer.

Funding All the authors declare no funding.

\section{Declarations}

Conflict of interest All the authors (David Groheux and Elif Hindié) declare no conflict of interest.
Human and animal studies This article does not contain any studies with human or animal subjects performed by the any of the authors.

\section{References}

1. Siegel RL, Miller KD, Fuchs HE, Jemal A (2021) Cancer statistics, 2021. CA Cancer J Clin 71:7-33

2. Salaün P-Y, Abgral R, Malard O, Querellou-Lefranc S, Quere G, Wartski M et al (2020) Good clinical practice recommendations for the use of PET/CT in oncology. Eur J Nucl Med Mol Imaging 47:28-50

3. Groheux D, Giacchetti S, Moretti J-L, Porcher R, Espié M, Lehmann-Che J et al (2011) Correlation of high (18)F-FDG uptake to clinical, pathological and biological prognostic factors in breast cancer. Eur J Nucl Med Mol Imaging 38:426-435

4. Buck A, Schirrmeister H, Kühn T, Shen C, Kalker T, Kotzerke J et al (2002) FDG uptake in breast cancer: correlation with biological and clinical prognostic parameters. Eur J Nucl Med Mol Imaging 29:1317-1323

5. Bos R, van Der Hoeven JJM, van Der Wall E, van Der Groep P, van Diest PJ, Comans EFI et al (2002) Biologic correlates of (18) fluorodeoxyglucose uptake in human breast cancer measured by positron emission tomography. J Clin Oncol Off J Am Soc Clin Oncol 20:379-387

6. Humbert O, Berriolo-Riedinger A, Cochet A, Gauthier M, Charon-Barra C, Guiu S et al (2014) Prognostic relevance at 5 years of the early monitoring of neoadjuvant chemotherapy using (18) F-FDG PET in luminal HER2-negative breast cancer. Eur J Nucl Med Mol Imaging 41:416-427

7. Crowe JP, Adler LP, Shenk RR, Sunshine J (1994) Positron emission tomography and breast masses: comparison with clinical, mammographic, and pathological findings. Ann Surg Oncol $1: 132-140$

8. Scheidhauer K, Scharl A, Pietrzyk U, Wagner R, Göhring UJ, Schomäcker K et al (1996) Qualitative [18F]FDG positron emission tomography in primary breast cancer: clinical relevance and practicability. Eur J Nucl Med 23:618-623

9. Schirrmeister H, Kühn T, Guhlmann A, Santjohanser C, Hörster T, Nüssle K et al (2001) Fluorine-18 2-deoxy-2-fluoro-D-glucose PET in the preoperative staging of breast cancer: comparison with the standard staging procedures. Eur J Nucl Med 28:351-358 
10. Samson DJ, Flamm CR, Pisano ED, Aronson N (2002) Should FDG PET be used to decide whether a patient with an abnormal mammogram or breast finding at physical examination should undergo biopsy? Acad Radiol 9:773-783

11. Walter C, Scheidhauer K, Scharl A, Goering U-J, Theissen P, Kugel $\mathrm{H}$ et al (2003) Clinical and diagnostic value of preoperative MR mammography and FDG-PET in suspicious breast lesions. Eur Radiol 13:1651-1656

12. Kumar R, Chauhan A, Zhuang H, Chandra P, Schnall M, Alavi A (2006) Clinicopathologic factors associated with false negative FDG-PET in primary breast cancer. Breast Cancer Res Treat 98:267-274

13. Uematsu T, Kasami M, Yuen S (2009) Comparison of FDG PET and MRI for evaluating the tumor extent of breast cancer and the impact of FDG PET on the systemic staging and prognosis of patients who are candidates for breast-conserving therapy. Breast Cancer Tokyo Jpn 16:97-104

14. Avril N, Rosé CA, Schelling M, Dose J, Kuhn W, Bense S et al (2000) Breast imaging with positron emission tomography and fluorine-18 fluorodeoxyglucose: use and limitations. J Clin Oncol Off J Am Soc Clin Oncol 18:3495-3502

15. Groheux D, Moretti J-L, Giacchetti S, Hindié E, Teyton P, Cuvier $\mathrm{C}$ et al (2009) PET/CT in breast cancer: an update. Bull Cancer (Paris) 96:1053-1070

16. Alberini J-L, Lerebours F, Wartski M, Fourme E, Le Stanc E, Gontier E et al (2009) 18F-fluorodeoxyglucose positron emission tomography/computed tomography (FDG-PET/CT) imaging in the staging and prognosis of inflammatory breast cancer. Cancer 115:5038-5047

17. Kumar R, Loving VA, Chauhan A, Zhuang H, Mitchell S, Alavi A (2005) Potential of dual-time-point imaging to improve breast cancer diagnosis with (18)F-FDG PET. J Nucl Med Off Publ Soc Nucl Med 46:1819-1824

18. Berg WA, Madsen KS, Schilling K, Tartar M, Pisano ED, Larsen LH et al (2011) Breast cancer: comparative effectiveness of positron emission mammography and MR imaging in presurgical planning for the ipsilateral breast. Radiology 258:59-72

19. Kalinyak JE, Berg WA, Schilling K, Madsen KS, Narayanan D, Tartar M (2013) Breast cancer detection using high-resolution breast PET compared to whole-body PET or PET/CT. Eur J Nucl Med Mol Imaging 41(2):260-275

20. Caldarella C, Treglia G, Giordano A (2014) Diagnostic performance of dedicated positron emission mammography using fluorine-18-fluorodeoxyglucose in women with suspicious breast lesions: a meta-analysis. Clin Breast Cancer 14:241-248

21. Ming Y, Wu N, Qian T, Li X, Wan DQ, Li C et al (2020) Progress and future trends in PET/CT and PET/MRI molecular imaging approaches for breast cancer. Front Oncol 10:1301

22. Taneja S, Jena A, Goel R, Sarin R, Kaul S (2014) Simultaneous whole-body (18)F-FDG PET-MRI in primary staging of breast cancer: a pilot study. Eur J Radiol 83(12):2231-2239

23. Litmanovich D, Gourevich K, Israel O, Gallimidi Z (2009) Unexpected foci of $18 \mathrm{~F}-\mathrm{FDG}$ uptake in the breast detected by PET/CT: incidence and clinical significance. Eur J Nucl Med Mol Imaging 36:1558-1564

24. Edge SB, Compton CC (2010) The American Joint Committee on Cancer: the 7th edition of the AJCC cancer staging manual and the future of TNM. Ann Surg Oncol 17:1471-1474

25. Heusner TA, Kuemmel S, Umutlu L, Koeninger A, Freudenberg LS, Hauth EAM et al (2008) Breast cancer staging in a single session: whole-body PET/CT mammography. J Nucl Med Off Publ Soc Nucl Med 49:1215-1222

26. Wahl RL, Siegel BA, Coleman RE, Gatsonis CG (2004) Prospective multicenter study of axillary nodal staging by positron emission tomography in breast cancer: a report of the staging breast cancer with PET Study Group. J Clin Oncol Off J Am Soc Clin Oncol 22:277-285

27. Veronesi U, De Cicco C, Galimberti VE, Fernandez JR, Rotmensz N, Viale G et al (2007) A comparative study on the value of FDG-PET and sentinel node biopsy to identify occult axillary metastases. Ann Oncol Off J Eur Soc Med Oncol ESMO 18:473-478

28. Pritchard KI, Julian JA, Holloway CMB, McCready D, Gulenchyn KY, George R et al (2012) Prospective study of 2-[ ${ }^{18}$ F $]$ fluorodeoxyglucose positron emission tomography in the assessment of regional nodal spread of disease in patients with breast cancer: an Ontario clinical oncology group study. J Clin Oncol Off J Am Soc Clin Oncol 30:1274-1279

29. Hindié E, Groheux D, Brenot-Rossi I, Rubello D, Moretti J-L, Espié M (2011) The sentinel node procedure in breast cancer: nuclear medicine as the starting point. J Nucl Med Off Publ Soc Nucl Med 52:405-414

30. Cooper KL, Harnan S, Meng Y, Ward SE, Fitzgerald P, Papaioannou D et al (2011) Positron emission tomography (PET) for assessment of axillary lymph node status in early breast cancer: a systematic review and meta-analysis. Eur J Surg Oncol J Eur Soc Surg Oncol Br Assoc Surg Oncol 37:187-198

31. Ueda S, Tsuda H, Asakawa H, Omata J, Fukatsu K, Kondo N et al (2008) Utility of 18F-fluoro-deoxyglucose emission tomography/ computed tomography fusion imaging (18F-FDG PET/CT) in combination with ultrasonography for axillary staging in primary breast cancer. BMC Cancer 8:165

32. Cooper KL, Meng Y, Harnan S, Ward SE, Fitzgerald P, Papaioannou D et al (2011) Positron emission tomography (PET) and magnetic resonance imaging (MRI) for the assessment of axillary lymph node metastases in early breast cancer: systematic review and economic evaluation. Health Technol Assess Winch Engl 15(iii-iv): $1-134$

33. Heusner TA, Kuemmel S, Hahn S, Koeninger A, Otterbach F, Hamami ME et al (2009) Diagnostic value of full-dose FDG PET/ CT for axillary lymph node staging in breast cancer patients. Eur J Nucl Med Mol Imaging 36:1543-1550

34. Yang WT, Le-Petross HT, Macapinlac H, Carkaci S, GonzalezAngulo AM, Dawood S et al (2008) Inflammatory breast cancer: PET/CT, MRI, mammography, and sonography findings. Breast Cancer Res Treat 109:417-426

35. van der Hoeven JJM, Krak NC, Hoekstra OS, Comans EFI, Boom RPA, van Geldere D et al (2004) 18F-2-fluoro-2-deoxyD-glucose positron emission tomography in staging of locally advanced breast cancer. J Clin Oncol Off J Am Soc Clin Oncol 22:1253-1259

36. Carkaci S, Macapinlac HA, Cristofanilli M, Mawlawi O, Rohren E, Gonzalez Angulo AM et al (2009) Retrospective study of 18FFDG PET/CT in the diagnosis of inflammatory breast cancer: preliminary data. J Nucl Med Off Publ Soc Nucl Med 50:231-238

37. Walker GV, Niikura N, Yang W, Rohren E, Valero V, Woodward WA et al (2012) Pretreatment staging positron emission tomography/computed tomography in patients with inflammatory breast cancer influences radiation treatment field designs. Int J Radiat Oncol Biol Phys 83:1381-1386

38. Groheux D, Giacchetti S, Delord M, Hindié E, Vercellino L, Cuvier C et al (2013) 18F-FDG PET/CT in staging patients with locally advanced or inflammatory breast cancer: comparison to conventional staging. J Nucl Med Off Publ Soc Nucl Med 54:5-11

39. van Uden DJP, Prins MW, Siesling S, de Wilt JHW, BlankenPeeters CFJM, Aarntzen EHJG (2020) [18F]FDG PET/CT in the staging of inflammatory breast cancer: a systematic review. Crit Rev Oncol Hematol 151:102943

40. NCCN Clinical Practice Guidelines in Oncology (2021) Breast Cancer. Version 1. http://www.nccn.org/professionals/physician_ gls/f_guidelines.asp. Assessed 5 Feb 2021 
41. Giuliano AE, Connolly JL, Edge SB, Mittendorf EA, Rugo HS, Solin LJ et al (2017) Breast Cancer-Major changes in the American Joint Committee on Cancer eighth edition cancer staging manual. CA Cancer J Clin 67:290-303

42. Groheux D, Moretti J-L, Baillet G, Espie M, Giacchetti S, Hindie E et al (2008) Effect of (18)F-FDG PET/CT imaging in patients with clinical Stage II and III breast cancer. Int J Radiat Oncol Biol Phys 71:695-704

43. Fuster D, Duch J, Paredes P, Velasco M, Muñoz M, Santamaría $\mathrm{G}$ et al (2008) Preoperative staging of large primary breast cancer with [18F]fluorodeoxyglucose positron emission tomography/ computed tomography compared with conventional imaging procedures. J Clin Oncol Off J Am Soc Clin Oncol 26:4746-4751

44. Segaert I, Mottaghy F, Ceyssens S, De Wever W, Stroobants S, Van Ongeval C et al (2010) Additional value of PET-CT in staging of clinical stage IIB and III breast cancer. Breast J 16:617-624

45. Aukema TS, Straver ME, Peeters M-JTFDV, Russell NS, Gilhuijs KGA, Vogel WV et al (2010) Detection of extra-axillary lymph node involvement with FDG PET/CT in patients with stage II-III breast cancer. Eur J Cancer Oxf Engl 1990 46:3205-3210

46. Koolen BB, Vrancken Peeters M-JTFD, Aukema TS, Vogel WV, Oldenburg HSA, van der Hage JA et al (2012) 18F-FDG PET/CT as a staging procedure in primary stage II and III breast cancer: comparison with conventional imaging techniques. Breast Cancer Res Treat 131:117-126

47. Groheux D, Giacchetti S, Espié M, Vercellino L, Hamy A-S, Delord $\mathrm{M}$ et al (2011) The yield of 18F-FDG PET/CT in patients with clinical stage IIA, IIB, or IIIA breast cancer: a prospective study. J Nucl Med Off Publ Soc Nucl Med 52:1526-1534

48. Groheux D, Hindié E, Delord M, Giacchetti S, Hamy A-S, de Bazelaire C et al (2012) Prognostic impact of 18FDG-PET-CT findings in clinical stage III and IIB breast cancer. J Natl Cancer Inst 104:1879-1887

49. Cochet A, Dygai-Cochet I, Riedinger J-M, Humbert O, BerrioloRiedinger A, Toubeau $\mathrm{M}$ et al (2014) ${ }^{18} \mathrm{~F}-\mathrm{FDG}$ PET/CT provides powerful prognostic stratification in the primary staging of large breast cancer when compared with conventional explorations. Eur J Nucl Med Mol Imaging 41:428-437

50. Jeong YJ, Kang D-Y, Yoon HJ, Son HJ (2014) Additional value of F-18 FDG PET/CT for initial staging in breast cancer with clinically negative axillary nodes. Breast Cancer Res Treat 145:137-142

51. Riedl CC, Slobod E, Jochelson M, Morrow M, Goldman DA, Gonen $\mathrm{M}$ et al (2014) Retrospective analysis of 18F-FDG PET/ CT for staging asymptomatic breast cancer patients younger than 40 years. J Nucl Med Off Publ Soc Nucl Med 55:1578-1583

52. Krammer J, Schnitzer A, Kaiser CG, Buesing KA, Sperk E, Brade $\mathrm{J}$ et al (2015) (18) F-FDG PET/CT for initial staging in breast cancer patients-Is there a relevant impact on treatment planning compared to conventional staging modalities? Eur Radiol 25(8):2460-2469

53. Evangelista L, Cervino AR, Michieletto S, Saibene T, Ghiotto C, Guarneri V et al (2017) Diagnostic and prognostic impact of fluorine-18-fluorodeoxyglucose PET/CT in preoperative and postoperative setting of breast cancer patients. Nucl Med Commun 38:537-545

54. Groheux D, Espié M, Giacchetti S, Hindié E (2013) Performance of FDG PET/CT in the clinical management of breast cancer. Radiology 266:388-405

55. Seo MJ, Lee JJ, Kim HO, Chae S-Y, Park SH, Ryu J-S et al (2014) Detection of internal mammary lymph node metastasis with (18) F-fluorodeoxyglucose positron emission tomography/computed tomography in patients with stage III breast cancer. Eur J Nucl Med Mol Imaging 41:438-445

56. Borm KJ, Voppichler J, Düsberg M, Oechsner M, Vag T, Weber W et al (2019) FDG/PET-CT-based lymph node atlas in breast cancer patients. Int J Radiat Oncol Biol Phys 103:574-582
57. Brown AH, Shah S, Groves AM, Wan S, Malhotra A (2021) The challenge of staging breast cancer with PET/CT in the era of COVID vaccination. Clin Nucl Med. https://doi.org/10.1097/ RLU.0000000000003683.

58. Morris PG, Lynch C, Feeney JN, Patil S, Howard J, Larson SM et al (2010) Integrated positron emission tomography/computed tomography may render bone scintigraphy unnecessary to investigate suspected metastatic breast cancer. J Clin Oncol Off J Am Soc Clin Oncol 28:3154-3159

59. Cook GJ, Houston S, Rubens R, Maisey MN, Fogelman I (1998) Detection of bone metastases in breast cancer by 18FDG PET: differing metabolic activity in osteoblastic and osteolytic lesions. J Clin Oncol Off J Am Soc Clin Oncol 16:3375-3379

60. Vogsen M, Jensen JD, Christensen IY, Gerke O, Jylling AMB, Larsen LB et al (2021) FDG-PET/CT in high-risk primary breast cancer-a prospective study of stage migration and clinical impact. Breast Cancer Res Treat 185(1):145-153

61. Ulaner GA, Castillo R, Wills J, Gönen M, Goldman DA (2017) 18F-FDG-PET/CT for systemic staging of patients with newly diagnosed ER-positive and HER2-positive breast cancer. Eur J Nucl Med Mol Imaging 44:1420-1427

62. Dashevsky BZ, Goldman DA, Parsons M, Gönen M, Corben AD, Jochelson MS et al (2015) Appearance of untreated bone metastases from breast cancer on FDG PET/CT: importance of histologic subtype. Eur J Nucl Med Mol Imaging 42:1666-1673

63. Hogan MP, Goldman DA, Dashevsky B, Riedl CC, Gönen M, Osborne JR et al (2015) Comparison of 18F-FDG PET/CT for systemic staging of newly diagnosed invasive lobular carcinoma versus invasive ductal carcinoma. J Nucl Med Off Publ Soc Nucl Med 56:1674-1680

64. Ulaner GA, Castillo R, Goldman DA, Wills J, Riedl CC, PinkerDomenig K et al (2016) (18)F-FDG-PET/CT for systemic staging of newly diagnosed triple-negative breast cancer. Eur J Nucl Med Mol Imaging 43:1937-1944

65. Lebon V, Alberini J-L, Pierga J-Y, Diéras V, Jehanno N, Wartski M (2017) Rate of distant metastases on 18F-FDG PET/CT at initial staging of breast cancer: comparison of women younger and older than 40 years. J Nucl Med Off Publ Soc Nucl Med $58: 252-257$

66. Srour MK, Amersi F (2020) Response to letter to the editor: "18FDG-PET/CT imaging in breast cancer patients with clinical stage IIB or higher.” Ann Surg Oncol 27:1710-1711

67. Urano M, Denewar FA, Murai T, Mizutani M, Kitase M, Ohashi $\mathrm{K}$ et al (2018) Internal mammary lymph node metastases in breast cancer: what should radiologists know? Jpn J Radiol 36:629-640

68. Hindié E, Groheux D, Hennequin C, Zanotti-Fregonara P, Vercellino L, Berenger N et al (2012) Lymphoscintigraphy can select breast cancer patients for internal mammary chain radiotherapy. Int J Radiat Oncol Biol Phys 83(4):1081-1088

69. Ko H, Baghdadi Y, Love C, Sparano JA (2020) Clinical utility of 18F-FDG PET/CT in staging localized breast cancer before initiating preoperative systemic therapy. J Natl Compr Cancer Netw JNCCN 18:1240-1246

70. Hyland CJ, Varghese F, Yau C, Beckwith H, Khoury K, Varnado $\mathrm{W}$ et al (2020) Use of 18F-FDG PET/CT as an initial staging procedure for stage II-III breast cancer: a multicenter value analysis. J Natl Compr Cancer Netw JNCCN 18:1510-1517

Publisher's Note Springer Nature remains neutral with regard to jurisdictional claims in published maps and institutional affiliations. 